Check for updates

Cite this: RSC Adv., 2018, 8, 11731

Received 5th February 2018

Accepted 21st March 2018

DOI: $10.1039 / \mathrm{c} 8 \mathrm{ra01125g}$

rsc.li/rsc-advances

\section{Purification, structure and anti-oxidation of polysaccharides from the fruit of Nitraria tangutorum Bobr.}

\begin{abstract}
Baotang Zhao, (D) *ab Jing Liu, ${ }^{\mathrm{b}}$ Xin Chen, ${ }^{\mathrm{b}} \mathrm{Ji}_{\text {Zhang }}{ }^{\mathrm{b}}$ and Junlong Wang ${ }^{\mathrm{b}}$
In this paper, polysaccharides were extracted from the fruits of Nitraria tangutorum Bobr. (NTWP) using a hot water extraction method and extraction conditions were optimized by RSM. The optimal conditions were determined as follows: extraction time $7 \mathrm{~h}$, extraction temperature $60{ }^{\circ} \mathrm{C}$, ratio of water to raw material $15: 1$, and with these conditions, the yield was $14.01 \pm 0.11 \%$. After purification using DEAE-cellulose column and Sephadex G-200 column, NTWP-II was successfully obtained. The results of GC-MS and SEC-LLS analysis suggested that monosaccharide composition of NTWP-II was composed of Rha, Ara, Man, Glc and Gal with the molar ratio of $1.14: 2.5: 3.00: 2.69: 5.28$ and $M_{w}, M_{w} / M_{n}$ and $R_{z}$ $2.29 \times 10^{5}, 1.32,15.22$. The detailed structure of NTWP-II was characterized by FT-IR, NMR. Based on these analyses, the structure of the repeating unit of NTWP-II was established.
\end{abstract}

\section{Introduction}

Oxidation is an important energy production process in organisms. However, it is well-known that free radicals, especially reactive oxygen species such as hydrogen peroxide, hydroxyl radicals and superoxide which generate during metabolism, could damage fatty acids, proteins, DNA and other macromolecules in our bodies and result in various diseases including cardiovascular diseases, neurodegenerative diseases, cancers and aging-related disorders. Recent papers prove that synthetic antioxidants are potential hazards in liver damage, carcinogenesis et al. and are restricted. ${ }^{1,2}$ Thus, it is very important to exploit natural antioxidants, especially those of plant origins. Constituents and crude numerous plant extracts have been recognized as natural antioxidants, with beneficial effects against free radicals in biological systems.

Nitraria (Zygophyllaceae) includes 15 species and only Nitraria tangutorum Bobr. grows in China. ${ }^{3}$ It is used to sand stabilization because of natural ability in withstanding wind and sand. ${ }^{4}$ In addition, the fruits and seeds of Nitraria tangutorum Bobr. were often used by local residents to treat ailments of the spleen and stomach, indigestion, neurasthenia and colds, and the leaves were used as an antispasmodic, antineuropathic, and antiarrhythmic agent in folk medicine.

Thus, there was a growing interest in the area of research on the positive effect of Nitraria tangutorum Bobr. on human health and other benefits. Recent studies focused on ingredients such

${ }^{a}$ College of Food Science and Engineering, Gansu Agricultural University, Lanzhou, 730070, China. E-mail: zhaobaotang@126.com; Fax: +86-0931-7631201

${ }^{b}$ Bioactive Products Engineering Research Center for Gansu Distinctive Plants, Northwest Normal University, Lanzhou 730070, China as seed oil, anthocyanins, tangutorine, polysaccharides et al. from their leaves, seeds, fruits and juice by-products. ${ }^{2-8}$ Some researches indicated that seed oil has been shown to improve immune response, reduce oxidants, and mitigate fatigue. ${ }^{4-6}$ Nine anthocyanins were separated from the seed oil of Nitraria tangutorum by subcritical fluid extraction. ${ }^{5}$ Cyanidin derivatives, the main components, has a good antioxidant. ${ }^{4}$ François' research showed that hydroalcoholic extract from the fruits of Nitraria sibirica Pall. induced vasorelaxation. ${ }^{9}$ From the leaves of Nitraria tangutorum Bobr., Duan and Liu isolated tangutorine (1) and identified its structure. ${ }^{6,7}$ The fruits of Nitraria tangutorum Bobr. were traditional medicinal food of Tibetans and used to alleviate fatigue caused by oxygen deficiency for thousands of years. Wei hua Ni' study indicated that water-soluble polysaccharides from the fruits of Nitraria tangutorum Bobr. in Tibetan plateau significantly exhibited anti-fatigue activities for the first time, through triglyceride mobilization during exercise and protecting corpuscular membrane by prevention of lipid oxidation via modifying several enzyme activities. ${ }^{8}$ Zhang et al. reported that the polysaccharides from Nitraria fruit was composed of mannose, rhamnose, galacturonic acid, glucose, galactose and arabinose with approximate molar ratios of $9.2: 3.3: 1.1: 1: 1.9: 2.3 .^{10} \mathrm{Ni}$ et al. isolated the polysaccharides (NTWP) from Nitraria tangutorum Bobr. by hot water extraction, purified by DEAE-cellulose ion exchange chromatography and found that NTWP was composed of mannose, rhamnose, glucuronicacid, galacturonic acid, glucose, galactose and arabinose with approximate molar ratios of $3.9: 1.8: 0.2: 3.3: 70.6: 7.6: 13.1 .^{8}$ Although previous studies have reported the structural properties of the polysaccharides from the fruits of Nitraria, the results of these studies were not consistent. To scan the structural 
characterization of polysaccharides from Nitraria tangutorum Bobr., we purified NTWP by DEAE-cellulose anion-exchange chromatography and Sephadex G-200 column, identified their structural features by GC-MS, SEC-LLS, FT-IR and NMR. Furthermore, we evaluated antioxidant activity of NTWP-II in vitro.

\section{Materials and methods}

\subsection{Plant materials}

The fruits of Nitraria tangutorum Bobr. were collected from Min qin in Gansu, China at October 2012. The fresh fruits were air-dried, crushed and kept in plastic bags at room temperature for use.

\subsection{Chemicals and reagents}

Pyridine, acetic anhydride, 1,1-diphenyl-2-picrylhydrazyl (DPPH), butyl hydroxy anisd (BHA), nitro blue tetrazolium (NBT), dihydronicotineamidadenine dinucleotide (NADH), phenazinemethosulfate (PMS), ethylene diamine tetraacetic acid (EDTA), trifluoroacetic acid (TFA) and thiobarbituric acid (TBA) were purchased from Sigma Aldrich (St. Louis, MO, USA), and DEAEcellulose 52 was purchased from the Pharmacia Co. (Upp-sala, Sweden). The solvents for size exclusion chromatography coupled with laser light scattering (SEC-LLS) and gas chromatography-mass spectrometry (GC-MS) were of chromatographic purification. All other reagents were of analytical grade.

\subsection{Preparation of NTWP}

The extraction process was carried out by the former report with slight modification. ${ }^{8}$ The dried fruit powder was defatted with petroleum ether, and refluxed with petroleum benzine to remove lipids, some colored ingredients and small molecular impurities. After removing the solvent, the resulting pretreated powder was dried and used for the extraction of NTWP. The pretreated samples were soaked in distilled water at room temperature. For hot water treatment, a sample $(1 \mathrm{~g})$ was put into a triangular flask (1 L), diluted with distilled water at a liquid-solid ratio varying from $8: 1$ to $25: 1$, v/w, and extracted at $30-80{ }^{\circ} \mathrm{C}$ for $1-12 \mathrm{~h}$. The mixture was centrifuged at $5000 \mathrm{rpm}$ for $15 \mathrm{~min}$, and the insoluble residue was retreated as mentioned above for 2 times. The supernatants were collected, concentrated by a rotary evaporator under reduced pressure to an appropriate volume, then the supernatant was deproteinized by using Sevage's method. Then the deproteinized supernatant was dialyzed, concentrated, mixed with four times of absolute ethanol and kept overnight at $4{ }^{\circ} \mathrm{C}$. The resulting precipitates were collected by centrifugation at $5000 \mathrm{rpm}$ for $10 \mathrm{~min}$, washed sequentially with anhydrous ethanol and acetone, and dried to afford crude NTWP. The extraction yield (\%) was calculated with the formula below:

$$
Y(\%)=\frac{W_{1}(\mathrm{~g})}{W_{0}(\mathrm{~g})}
$$

where $Y$ was the yield of polysaccharide (\%), $W_{1}$ was the polysaccharides of extraction (g), and $W_{0}$ represented dried sample weight (g).

\subsection{Experimental design of NTWP}

Based on the results of single factor experiments, a three-level BBD with three factors was applied to determine the optimal levels for extraction temperature $\left(X_{1}\right)$, extraction time $\left(X_{2}\right)$ and ratio of water to raw material $\left(X_{3}\right)$, which significantly affected the extraction efficiency, and the optimal range of each variable was determined. The experimental designs of the code and the actual levels of each factor are presented in Table 1. As shown in Table 1 , the three factors chosen for this study were designated as $X_{1}, X_{2}$ and $X_{3}$ and were prescribed into three levels, coded +1 , 0 , and -1 for high, intermediate and low value, respectively.

Test variables were coded according to the following equation:

$$
x_{i}=\frac{X_{i}-X_{0}}{\Delta X}
$$

where $x_{i}$ was the coded value of an independent variable; $X_{i}$ was the actual value of an independent variable; $X_{0}$ was the actual value of an independent variable at centre point; $\Delta X$ was the step change value of an independent variable. The whole design consisted of 17 experimental points carried out in random order (Table 2).

All experiments were performed in triplicate and the averages of polysaccharide yields were taken as response. For predicting the optimal point, a second order polynomial model was fitted to correlate relationship between independent variables and response (polysaccharide yield). For the three factors, the equation was:

$$
Y=A_{0}+\sum A_{i x} i+\sum A_{i i} X_{i 2}+\sum A_{i j} X_{i} X_{j}
$$

where $Y$ was the response variables (yields of polysaccharides in real values). $A_{0}, A_{i}, A_{i i}, A_{i j}$ were the regression coefficients of variables for intercept, linear, quadratic and interaction terms, respectively. $X_{i}$ and $X_{j}$ were independent variables $(i \neq j)$.

Table 1 Independent variables and their levels used in the response surface design ${ }^{a}$

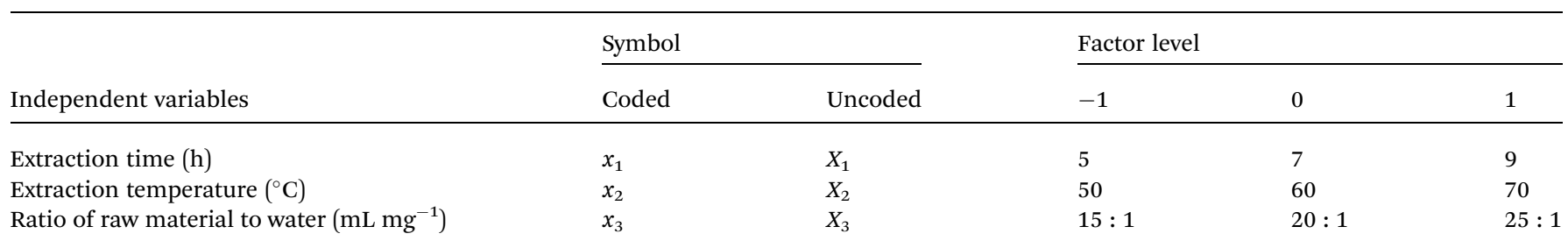

${ }^{a} x_{1}=\left(X_{1}-7\right) / 2 ; x_{2}=\left(X_{2}-60\right) / 10 ; x_{3}=\left(X_{3}-20\right) / 5$. 
Table 2 Experimental results of the response surface methodology

\begin{tabular}{|c|c|c|c|c|}
\hline No. & $\begin{array}{l}X_{1} \text { extraction } \\
\text { 4time }(\mathrm{h})\end{array}$ & $\begin{array}{l}X_{2}: \text { extraction } \\
\text { temperature }\left({ }^{\circ} \mathrm{C}\right)\end{array}$ & $\begin{array}{l}X_{3}: \text { ratio of water } \\
\text { to material }\left(\mathrm{mL} \mathrm{g}^{-1}\right)\end{array}$ & $\begin{array}{l}\text { Y: extraction } \\
\operatorname{yield}^{a}(\%)\end{array}$ \\
\hline 1 & -1 & -1 & 0 & 12.015 \\
\hline 2 & 1 & -1 & 0 & 12.09 \\
\hline 3 & -1 & 1 & 0 & 12.405 \\
\hline 4 & 1 & 1 & 0 & 12.75 \\
\hline 5 & -1 & 0 & -1 & 11.46 \\
\hline 6 & 1 & 0 & -1 & 10.885 \\
\hline 7 & -1 & 0 & 1 & 11.745 \\
\hline 8 & 1 & 0 & 1 & 12.21 \\
\hline 9 & 0 & -1 & -1 & 9.885 \\
\hline 10 & 0 & 1 & -1 & 12.51 \\
\hline 11 & 0 & -1 & 1 & 12.91 \\
\hline 12 & 0 & 1 & 1 & 10.805 \\
\hline 13 & 0 & 0 & 0 & 14.19 \\
\hline 14 & 0 & 0 & 0 & 14.04 \\
\hline 15 & 0 & 0 & 0 & 14.475 \\
\hline 16 & 0 & 0 & 0 & 13.95 \\
\hline 17 & 0 & 0 & 0 & 13.875 \\
\hline
\end{tabular}

The significance in the model was evaluated by analysis of variance (ANOVA). The accuracy and general ability of the polynomial model could be evaluated by a determination coefficient $R^{2}$ and adjusted coefficient of determination $R_{\mathrm{adj}}{ }^{2}$. Subsequently, several confirmation experiments were conducted to verify the validity of the statistical experimental strategies. The regression coefficients were then used to make statistical calculation to generate dimensional and contour maps from the regression models. Statistica (Version 8.0, USA) software package was used to analyze the experimental data. $P$-values of less than 0.05 were considered to be statistically significant.

\subsection{Isolation and purification of NTWP}

The crude polysaccharide (NTWP), obtained under the optimal conditions, was redissolved in distilled water, and the supernatant was loaded onto a DEAE-cellulose column $(2.6 \mathrm{~cm} \times 50 \mathrm{~cm})$, eluting stepwise with a gradient of aqueous solution of sodium chloride $(\mathrm{NaCl}, 0-1.0 \mathrm{M})$ at a flow rate of $0.2 \mathrm{~mL} \mathrm{~min}^{-1}$. The eluate was collected by using an automated fraction collector. In order to detect polysaccharides, a $0.2 \mathrm{~mL}$ sample collected from each eluted fraction ( $2 \mathrm{~mL}$ per tube) was mixed with sulfuric acid and phenol to produce color reaction. The carbohydrate content of each tube was monitored at $490 \mathrm{~nm}$. The fractions with rose color were combined, concentrated, dialyzed, and lyophilized, after which the main polysaccharide was redissolved in deionized water and loaded onto a Sephadex G-200 column $(20 \times 500 \mathrm{~mm})$. After loading the sample onto the column, the column was eluted with deionized water at a flow rate of $0.5 \mathrm{~mL} \mathrm{~min}^{-1}$. The main polysaccharide fractions were collected, combined, and lyophilized to obtain a purified polysaccharide (NTWP-II).

\subsection{Structural characterization of NTWP-II}

2.6.1. Components analysis. The carbohydrate and proteins contents of NTWP-II were determined by the phenol- sulphuric acid method and Bradford's method according to the method by our report. The composition was analyzed according to the earlier report from our laboratory. ${ }^{\mathbf{1 1}}$ Briefly, $4 \mathrm{mg}$ of sample were dissolved in $4 \mathrm{~mL}$ of $4 \mathrm{M}$ trifluoroacetic acid acetate (TFA) in a test tube and then hydrolysed at $120{ }^{\circ} \mathrm{C}$ for $10 \mathrm{~h}$ under air-tight conditions. TFA was then evaporated through decompression and distillation. When the tube was dry, $10 \mathrm{mg}$ of ammonium hydrochloride and $0.5 \mathrm{mg}$ pyridine were added and allowed to react in a $90{ }^{\circ} \mathrm{C}$ water bath for $30 \mathrm{~min}$. Then $0.5 \mathrm{~mL}$ of cold (kept at $4{ }^{\circ} \mathrm{C}$ in a refrigerator) acetic anhydride was added to the test tube and the mixture was incubated in the $90{ }^{\circ} \mathrm{C}$ water bath for a further $30 \mathrm{~min}$ to allow the acetylation reaction to occur. The end-product was decompressed and distilled to dryness. The acetate derivatives were analysed by GC-MS with an HP-5 capillary column (HP 6820, Hewlett-Packard). The temperature programme was set to increase from $120{ }^{\circ} \mathrm{C}$ to $250{ }^{\circ} \mathrm{C}$ with an increment of $5{ }^{\circ} \mathrm{C} \mathrm{min}{ }^{-1}$ and He was the carrier gas. The standard monosaccharides were measured following the same procedure. D-Amr, L-Rha, D-Lyx, DAra, D-Xyl, D-Man, D-Glu, D-Gal were used as references.

2.6.2. FT-IR analysis. The sample was ground with $\mathrm{KBr}$ powder and then pressed into pellets for Nicolet NEXUS 670 FTIR in the frequency range of $4000-400 \mathrm{~cm}^{-1}$ to detect functional groups. Sixteen scans at a resolution of $4 \mathrm{~cm}^{-1}$ were averaged and referenced against air.

2.6.3. Molecular weight determination. Size exclusion chromatography, combined with laser light scattering (SECLLS), measurements were carried out with a multi-angle laser photometer (MALLS, $k=690 \mathrm{~nm}$; DAWN EOS, WyattTechnology Co., USA). An UltrahydrogelTM column $(7.8 \times 300 \mathrm{~mm}$, Waters, USA) was used as the SEC instrument. An Optilab refractometer (Dawn, Wyatt Technology Co., USA) was simultaneously connected. The polysaccharide solutions with desired concentrations were prepared, and optical clarification of the solutions was achieved by filtration into a scattering cell. The injection

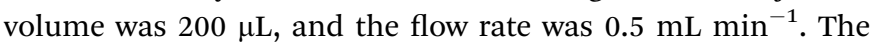
refractive index increment $(\mathrm{d} n / \mathrm{d} c)$ value of the sample was determined, by using an Optilab refractometer at $690 \mathrm{~nm}$ and $25^{\circ} \mathrm{C}$, to be $0.147 \mathrm{~mL} \mathrm{~g}^{-1}$. The basic light scattering equation is as follows:

$$
\frac{K_{\mathrm{C}}}{R_{\theta}}=\frac{1}{M}\left(1+\frac{16 \pi^{2}\left(S^{2}\right) z}{3 \lambda^{2}} \sin ^{2}\left(\frac{\theta}{2}\right)\right)+2 A_{2} C
$$

where, $K$ is an optical constant equal to $\left[4 \pi^{2} n^{2}(\mathrm{~d} n / \mathrm{d} c)\right] /\left(\lambda^{4} N_{\mathrm{A}}\right), c$ is the polysaccharide concentration in $\mathrm{mg} \mathrm{mL}^{-1}, R_{\theta}$ is the Rayleigh ratio, $\lambda$ is the wavelength, $n$ is the refractive index of the solvent, $\mathrm{d} n / \mathrm{d} c$ is the refractive index increment, $N_{\mathrm{A}}$, the Avogadro number, $N_{\mathrm{A}}$ is the second virial coefficient. As the column separates the polymer according to molecular weight, each fraction was led to the light scattering detector for instantaneous measurement of the scattering intensities. The refractive index detector, connected in series, gave the estimate of the polymer concentration. In chromatography mode, we have a single and sufficiently low concentration at a particular slice because of the further dilution by the SEC column of the already dilute injected solutions. 
a

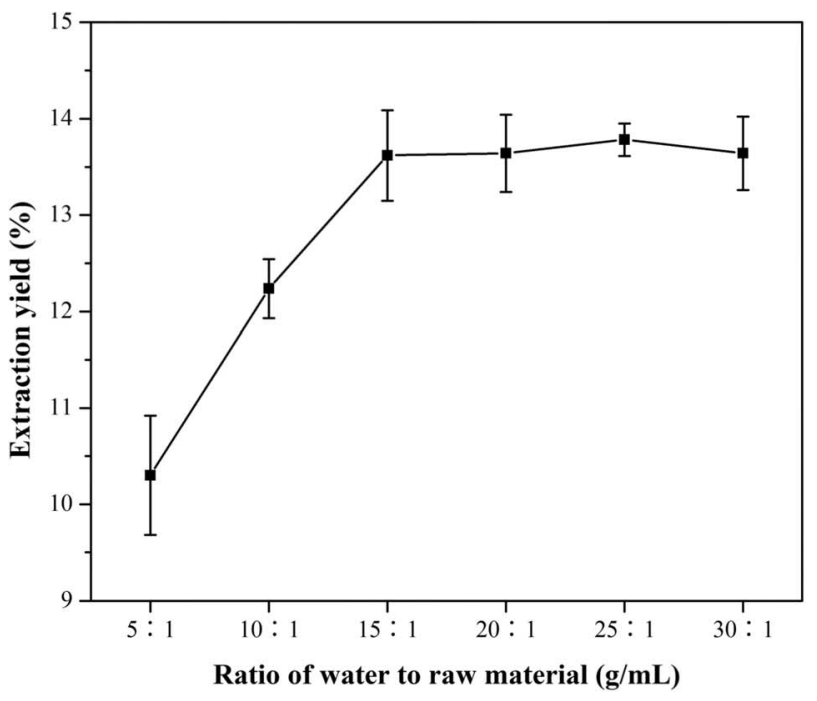

b

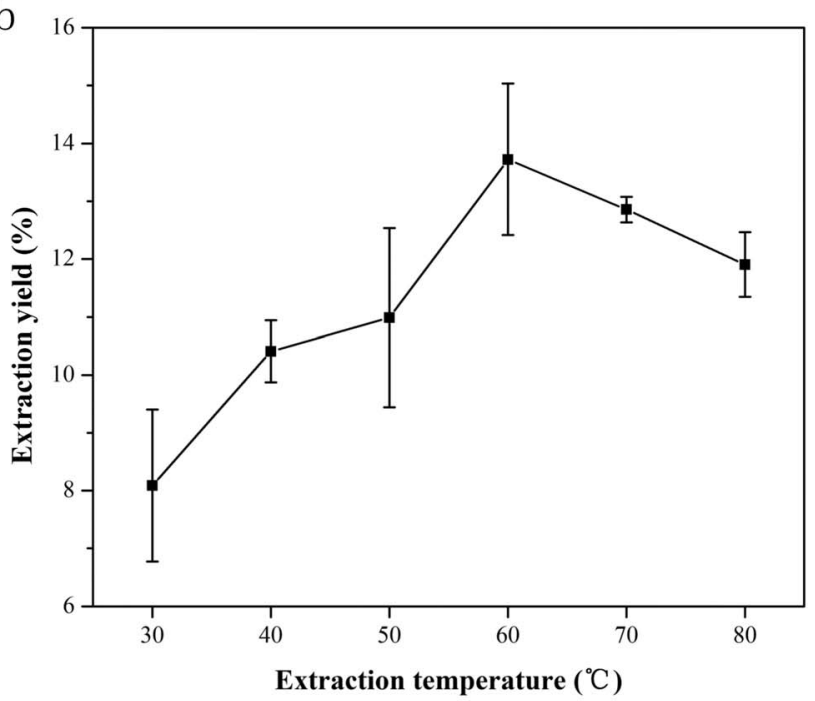

C

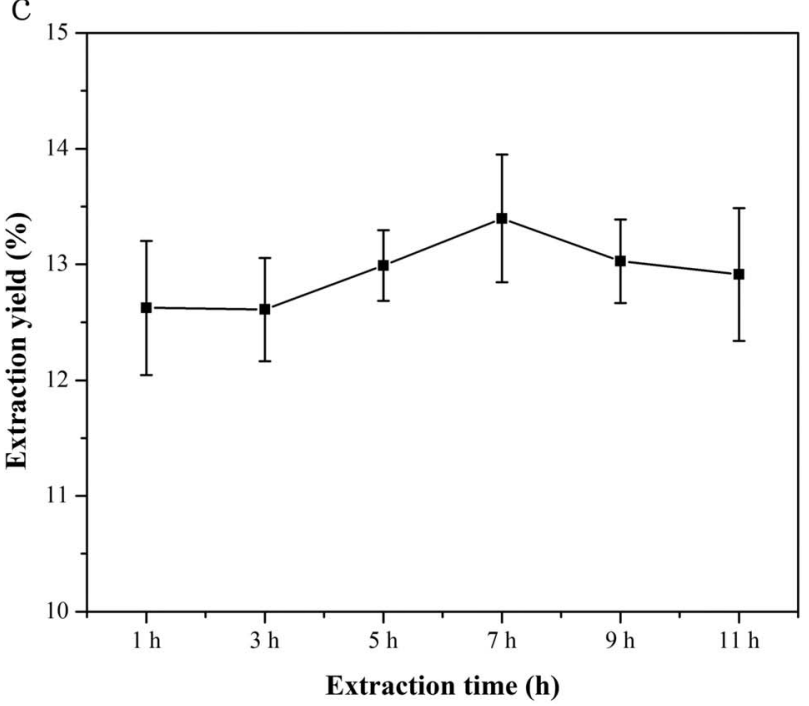

Fig. 1 Effect of ratio of water to raw material, extraction time and extraction temperature $\left((a)\right.$ extraction temperature $55^{\circ} \mathrm{C}$, extraction time $5 \mathrm{~h}$; (b) extraction time $5 \mathrm{~h}$, ratio of water to raw material $40: 1$ (v/ w); (c) ratio of water to raw material $40: 1(\mathrm{v} / \mathrm{w})$, extraction temperature $\left.55^{\circ} \mathrm{C}\right)$. Data are shown as mean $\pm \mathrm{SD}(n=3)$.
2.6.4. NMR spectroscopy. NMR spectra were recorded with a BRUKER400 $\mathrm{MHz}$ spectrometer at a probe temperature of $80{ }^{\circ} \mathrm{C}$. All exchangeable $\mathrm{H}$ of the samples were replaced by $\mathrm{D}_{2} \mathrm{O}$ before obtaining the spectra in deuterium oxide. Chemical shifts were calibrated using coaxial NMR tubes with (3trimethylsilyl)-propane sulfonic acid sodium salt (DSS, $\mathrm{dH}=$ $0.00, \mathrm{dC}=0.00)$ in the inner tube. The $2 \mathrm{D}^{1} \mathrm{H}^{-}{ }^{1} \mathrm{H}$ correlated spectroscopy (COSY), ${ }^{1} \mathrm{H}^{-1} \mathrm{H}$ total correlation spectroscopy (TOCSY), ${ }^{1} \mathrm{H}^{13} \mathrm{C}$ heteronuclear single quantum coherence (HMQC) and ${ }^{1} \mathrm{H}^{-13} \mathrm{C}$ heteronuclear multiple quantum coherence (HMBC) measurements were used to assign signals and to determine the sequence of sugar residues. ${ }^{12}$

\subsection{Assay for antioxidant activities}

Polysaccharides were dissolved in deionized water at the concentration of $0.02-5 \mathrm{mg} \mathrm{mL}^{-1}$. Antioxidant assay in vitro was carried out on scavenging DPPH, hydroxyl, superoxide radical and chelating metal, using $\mathrm{V}_{\mathrm{E}}, \mathrm{V}_{\mathrm{C}}$, BHT and EDTA as the positive controls according to the earlier report. ${ }^{11}$ Each concentration was measured in triplicate and averaged.

\section{Results and discussion}

\subsection{Influence of three single factors on the extraction efficiency of NTWP}

3.1.1. Effect of ratio of water to raw material on extraction yield of NTWP. Ratio of water to raw material was a routine parameter that significantly affects the extraction efficiency. In the present study, extraction of NTWP was carried out at different ratios of water to raw material $(5,10,15,20,25,30 \mathrm{~mL}$ $\mathrm{g}^{-1}$ ) when other parameters were as follows: extraction temperature $55{ }^{\circ} \mathrm{C}$ and extraction time $5 \mathrm{~h}$. As shown in Fig. 1a, the extraction yield increased with the increase of ratio of water to raw material from 5 to $15 \mathrm{~mL} \mathrm{~g}^{-1}$ and while above $15 \mathrm{~mL} \mathrm{~g}^{-1}$, the yield increased slowly up to its maximum amount of $13.42 \pm$ $0.4 \%$ at $20 \mathrm{~mL} \mathrm{~g}^{-1}$ when ratio of water to raw material continued to rise. This phenomenon could be explained that the higher the ratio of water to raw material was, the lower the concentration and viscosity of the extraction solvent would be. Hence more polysaccharides molecules could dissolve in water and the extraction yield increased. ${ }^{13}$ But the amount of polysaccharides in raw material was definite and further increase of ratio of water to material would not increase the extraction yield. Thus, ratio of water to raw material range of $15: 1$ to $25: 1$ was favorable for extracting NTWP.

3.1.2. Effect of extraction temperature on extraction yield of NTWP. Extraction time is another factor that influenced the extraction efficiency. To investigate the effect of temperature on extraction yield of NTWP, extraction was conducted at different temperatures $\left(30,40,50,60,70\right.$ and $80{ }^{\circ} \mathrm{C}$, respectively), when the other extraction variables were set as follows: ratio of water to raw material $20 \mathrm{~mL} \mathrm{~g}^{-1}$ and extraction time $5 \mathrm{~h}$. The effect of extraction time on the yield is presented in Fig. 1b. As shown in Fig. 1b, the extraction yield increased when temperature increased from 30 to $60{ }^{\circ} \mathrm{C}$, reaching a maximum $(13.30 \pm 0.6 \%)$ at $60{ }^{\circ} \mathrm{C}$, and then declined when extraction temperature continued to rise. 
Table 3 Analysis of variance of the experimental results of the BBD

\begin{tabular}{lcclrl}
\hline Variables & Sum of squares & DF & Mean square & $F$ value & $P$ value \\
\hline Model & 27.51 & 9 & 3.06 & 69.92 & $<0.0001$ \\
$X_{1}$ & 0.012 & 1 & 0.012 & 0.27 & 0.6163 \\
$X_{2}$ & 0.31 & 1 & 0.31 & 7.05 & 0.0327 \\
$X_{3}$ & 1.07 & 1 & 1.07 & 24.55 & 0.0016 \\
$X_{1} X_{2}$ & 0.018 & 1 & 0.018 & 0.42 & 0.5391 \\
$X_{1} X_{3}$ & 0.27 & 1 & 0.27 & 6.19 & 0.0418 \\
$X_{2} X_{3}$ & 5.59 & 1 & 5.59 & 127.95 & $<0.0001$ \\
$X_{1}{ }^{2}$ & 3.20 & 1 & 3.20 & 73.20 & $<0.0001$ \\
$X_{2}{ }^{2}$ & 3.56 & 1 & 3.56 & 81.39 & $<0.0001$ \\
$X_{3}{ }^{2}$ & 11.59 & 1 & 11.59 & 265.17 & $<0.0001$ \\
Residual & 0.31 & 7 & 0.044 & & \\
Lack of fit & 0.081 & 3 & 0.027 & 0.48 & 0.7148 \\
Pure error & 0.23 & 4 & 0.056 & & \\
& & & & &
\end{tabular}

Therefore, it could be explained that as the temperature increased, the polysaccharides diffusion coefficient would increase, resulting in an enhanced solubility of the polysaccharides in the solvent. However, when temperature became higher, the extraction yield of NTWP decreased drastically, probably due to that high temperature could destroy the structure of polysaccharides and lead to degradation. Therefore, extraction temperature range of $50-70{ }^{\circ} \mathrm{C}$ was favorable for extraction of NTWP, being selected for further optimization in BBD design.

3.1.3. Effect of extraction time on extraction yield of NTWP. To investigate the influence of time on extraction efficiency, extraction process was carried out at 1, 3, 5, 7, 9, $11 \mathrm{~h}$, while other parameters were as follows: ratio of water to raw material $20 \mathrm{~mL} \mathrm{~g}^{-1}$ and extraction temperature $60^{\circ} \mathrm{C}$. The effect of extraction time on the yield was presented in Fig. 1c. When extraction time varied from 1 to $9 \mathrm{~h}$, the variance of extraction yield was relatively rapid and reached the maximum extraction yield $(13.72 \pm 1.3 \%)$ at $7 \mathrm{~h}$, and then decreased as the extraction proceeded. These results can be explained that extended extraction time $(>7 \mathrm{~h})$ would result in the degradation of the polysaccharides, which was induced by their thermal instability. Therefore, after the maximum extraction yield was achieved, longer time of how water extraction was not necessary. Thus, temperature range of 5-7 h was selected as optimal in the BBD experiment.

\subsection{Optimization of extraction conditions of NTWP by BBD}

3.2.1. Predicted model and statistical analysis. As shown in Table 1, each experiment in the design matrix was performed and the experimental data were obtained. By applying multiple regression analysis on the experimental data, the Design-Expert software generated a second order polynomial equation that could express the relationship between process variables and the response. The final equation obtained in terms of coded factors was given below:

$$
\begin{aligned}
Y= & 14.11+0.039 X_{1}+0.2 X_{2}+0.37 X_{3}+0.067 X_{1} X_{2}+ \\
& 0.26 X_{1} X_{3}-1.18 X_{2} X_{3}-0.87 X_{1}^{2}-0.92 X_{2}^{2}-1.66 X_{3}^{2}
\end{aligned}
$$

where $Y$ was the predicted yield of polysaccharides, $X_{1}$ was ratio of water to raw material $(\mathrm{W}), X_{2}$ was extraction temperature $\left({ }^{\circ} \mathrm{C}\right)$ and $X_{3}$ was extraction time $(\mathrm{h})$.
Analysis of variance (ANOVA) was performed to evaluate the predictive model and the variables. The $P$-values was used as a tool to check the significance of each coefficient and indicated the pattern of interactions between variables. The smaller the value of $P$ was, the more significant the corresponding coefficient would be. A significant lack of fit demonstrated that the fitted model failed to failed to represent the data in the experimental domain at which points were not included in the regression. The ANOVA for the fitted quadratic polynomial model of extraction efficiency was presented in Table 3. As shown in Table 3, the quadratic regression model has a very low $P$-value $(P<0.0001)$, indicating that the fitness of the model was highly significant. The "Model $F$-Value" of 69.92 implied the model was significant. There was only a $0.01 \%(P<0.0001)$ chance that a "Model $F$-Value" could occur due to noise. At the same time, the "Lack of Fit F-Value" of 0.48 implied the lack of fit was not significant relative to the pure error. A "Lack of Fit $F$ Value" this large has $71.48 \%(P=0.7148)$ chance to occur because of noise. Coefficient of determination $\left(R^{2}\right)$ indicated that $98.90 \%$ of the variations can be explained by the fitted model. The value of adjusted determination coefficient $\left(R_{\mathrm{adj}}{ }^{2}\right)$ was 0.9749 , which also confirmed that the model was highly significant. Moreover, a relatively low value of coefficient variation $(1.68 \%)$ indicated high degree of precision and good deal of reliability for the experimental values. "Adeq Precision" measured the signal to noise ratio. A ratio greater than 4 was desirable. The ratio of 26.961 indicated an adequate signal. Thus, the results indicated that this model could be used to navigate the design space.

The significance of each coefficient was also determined by using $F$-value and $P$-value. It could be seen that the linear coefficients $\left(X_{2}\right.$ and $\left.X_{3}\right)$, quadratic terms coefficients $\left(X_{1}{ }^{2}, X_{2}{ }^{2}\right.$, and $\left.X_{3}{ }^{2}\right)$ and cross product coefficients $\left(X_{1} X_{3}, X_{2} X_{3}\right)$ were significant, with small $P$-values $(P<0.05)$. And the significant interaction between factors means that effect of them on the extraction yield of polysaccharides was not simply an additive. The empirical model was converted to three-dimensional (3D) and contour plots to predict the relationships between the independent variables and the response. ${ }^{\mathbf{1 4 , 1 5}}$

3.2.2. Optimization of extraction procedure. The graphical representation of regression equation was obtained using Design-Expert to evaluate the effects of independent variables and their interactions on extraction efficiency of NTWP. As graphical representations of the regression equation, three dimensional response surface and two dimensional contour plots were very useful to visualize the relationship between independent and dependent variables and the interactions between two variables. Different shapes of the contour plots indicated different interactions between the variables. Circular contour plot means the interactions between the corresponding variables are negligible, while elliptical contour suggested the interactions between the corresponding variables are significant. Among these three variables (ratio of water to raw material, extraction temperature and extraction time) as shown in Fig. 2, one variable was kept constant at zero level, when the other two variables within the experimental range were depicted in the plots. 
A

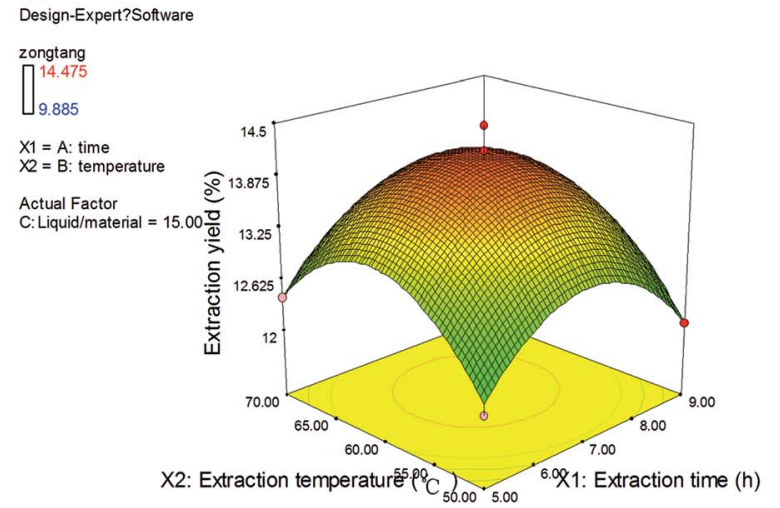

B

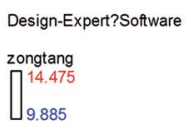

$X 1=A:$ time
$X 2=C$ : Liquid/ $X_{2}=C:$ Liquid/material Actual Factor B: temperature $=60.00$

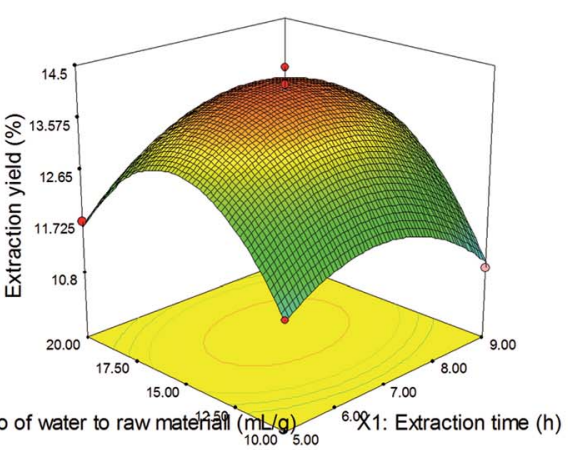

C

Design-Expert?Software

zongtang
$\prod_{9.885}^{14.475}$ $\mathrm{X} 1=\mathrm{B}:$ temperature
$\mathrm{X} 2=\mathrm{C}:$ Liquid/material Actual Factor A: time $=7.00$

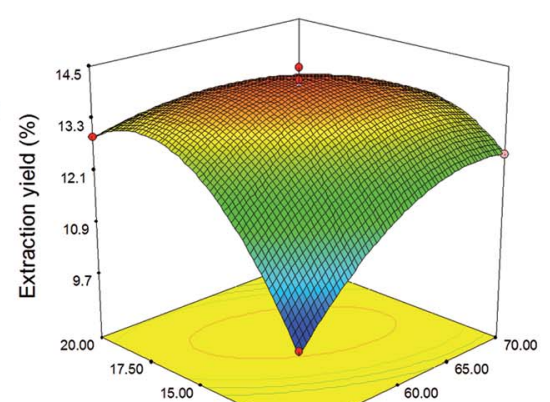

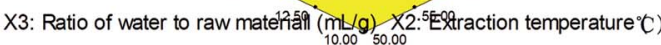

a

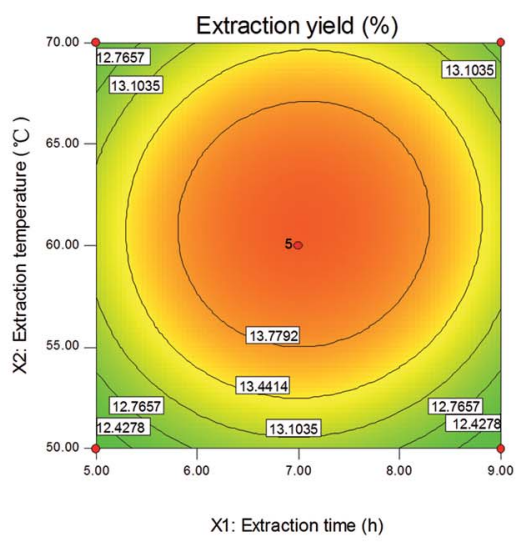

$\mathrm{b}$

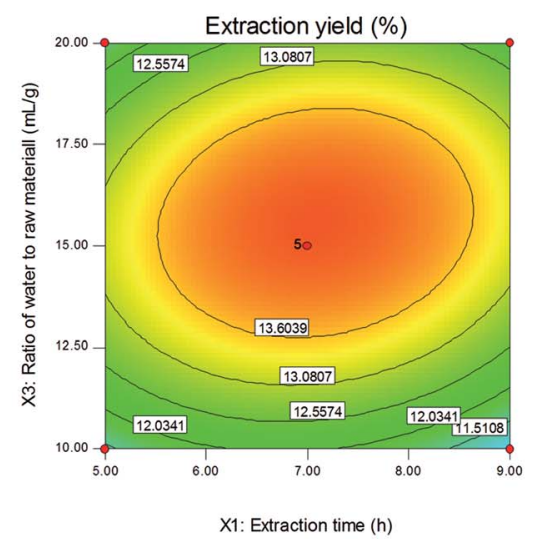

C

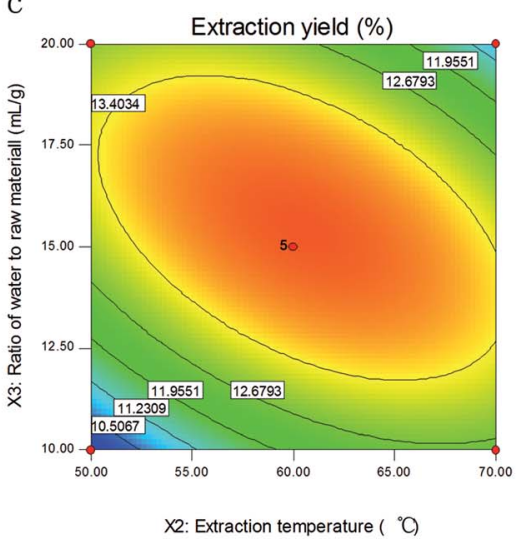

Fig. 2 Tri-dimensional response surface and contour plots showing the experimental factors and their mutual interactions on NTWP-II extraction ((a) extraction time and extraction temperature, (b) extraction time and ratio of water to raw material, (c) extraction temperature and ratio of water to raw material).

The extraction yield $(Y)$ of NTWP affected by extraction time $\left(X_{1}\right)$ and extraction temperature $\left(X_{2}\right)$ was shown in Fig. $2 \mathrm{~A}$ and a with ratio of water to raw material $\left(X_{3}\right)$ fixed at a zero level. The extraction yield $(Y)$ increases rapidly when ratio of extraction time $\left(X_{1}\right)$ and extraction temperature $\left(X_{2}\right)$ increase in the range of 5-6.8 $\mathrm{h}$ and $50-60{ }^{\circ} \mathrm{C}$, respectively; but beyond $6.8 \mathrm{~h}$ and $60{ }^{\circ} \mathrm{C}$, extraction yield $(Y)$ decreases slightly. The elliptical contour plot shown in Fig. 2A, a indicated the mutual interactions between extraction time $\left(X_{1}\right)$ and extraction temperature $\left(X_{2}\right)$ were significant.
Fig. 2B, b showed the 3D response surface plot and the 2D contour plot at varying extraction time and ratio of water to raw material at fixed extraction temperature (0 level). The same trends with Fig. 2A, a were depicted in Fig. 2B, b, of which Fig. 2B, b showed a similar elliptical contour plot. The extraction yield of polysaccharides affected by extraction temperature and ratio of water to raw material is shown in Fig. 2C, c with extraction time fixed at a zero level $(7 \mathrm{~h})$. The yield of polysaccharides increased evidently with the extraction temperature 

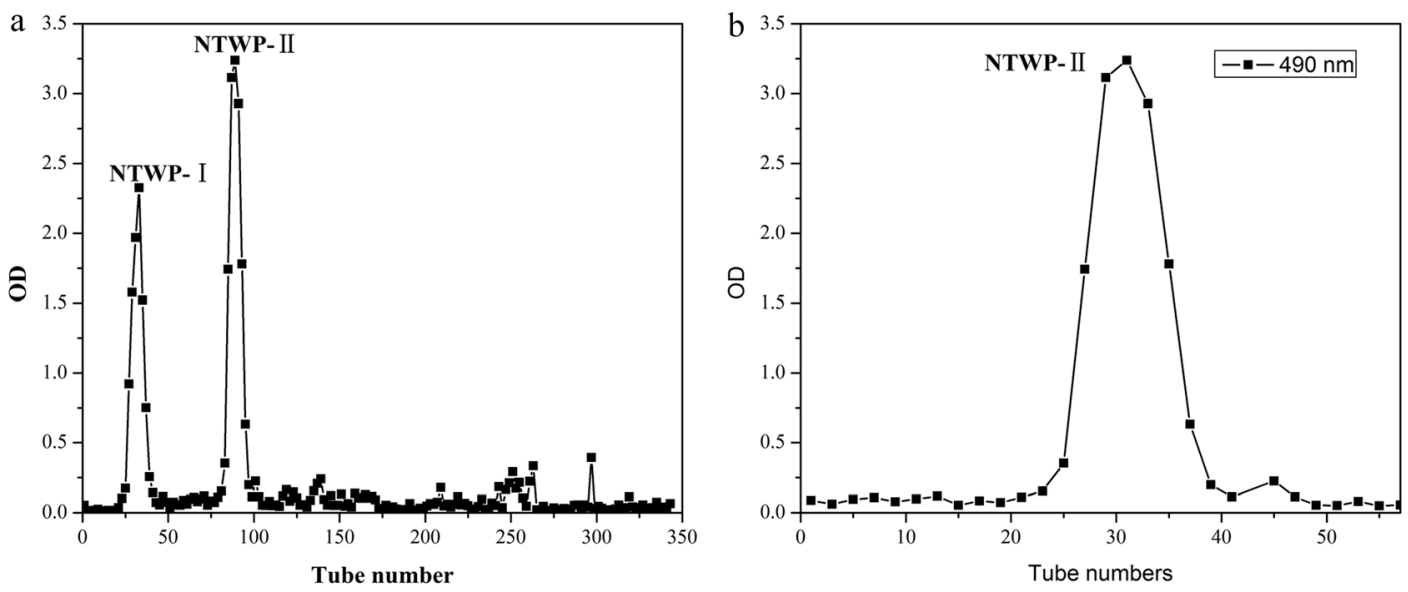

Fig. 3 Elution curve of NTWP on DEAE-cellulose column (a) and elution curves of NTWP-II on Sephadex G-200 column (b).
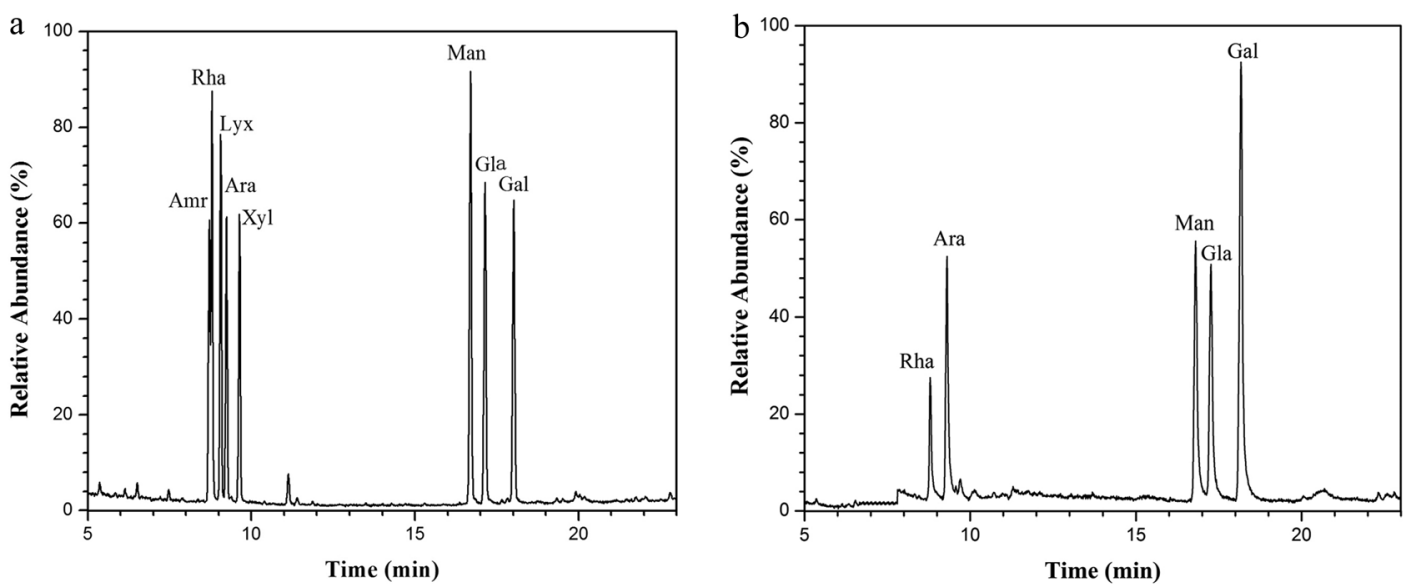

Fig. 4 The GC-MS chromatograms of derivatives of 8 standard monosaccharides (a) and component monosaccharides released from NTWP-II (b). Peaks: (1) D-Amr, (2) L-Rha, (3) D-Lyx, (4) D-Ara, (5) D-Xyl, (6) D-Man, (7) D-Gla, (8) D-Gal.

from $50{ }^{\circ} \mathrm{C}$ to $65{ }^{\circ} \mathrm{C}$. However, beyond $65^{\circ} \mathrm{C}$, the extraction yield would not increase as the temperature ascended.

3.2.3. Verification of predictive model. By employing the software Design-Expert, the solved optimum values of the tested variables were extraction time $7.08 \mathrm{~h}$, extraction temperature
$60.45{ }^{\circ} \mathrm{C}$, liquid-solid ratio $15: 49$. Under the optimal conditions, the maximum predicted yield of NTWP was $14.13 \%$. Taking account of the operating convenience, the optimal parameters were determined as following: extraction time $7 \mathrm{~h}$, extraction temperature $60{ }^{\circ} \mathrm{C}$, liquid-solid ratio $15: 1$.
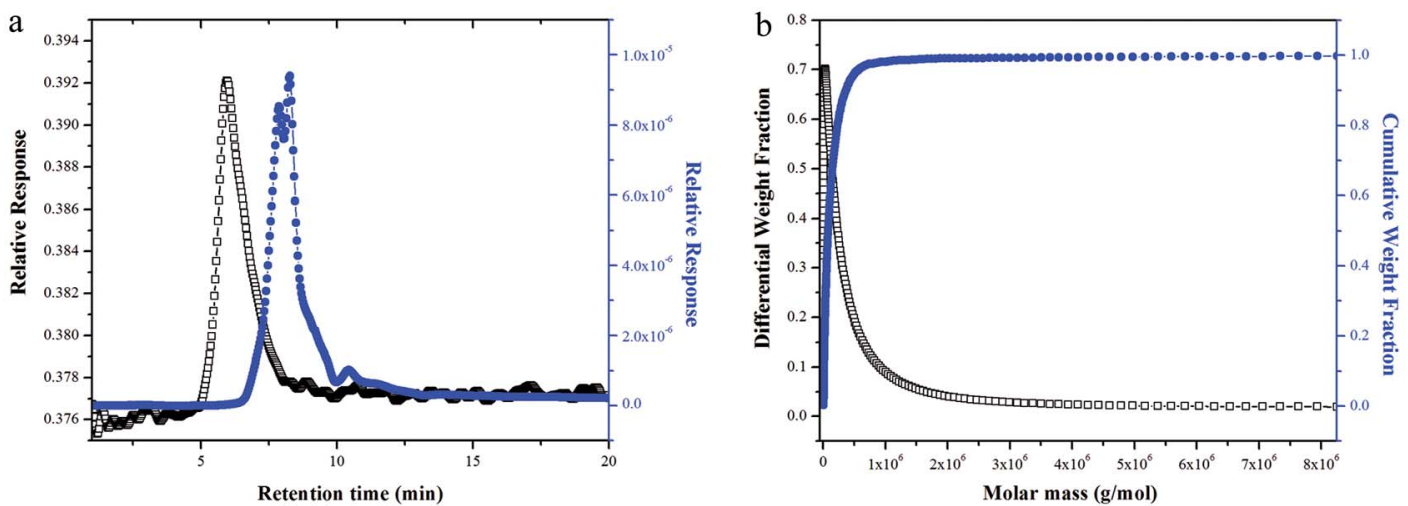

Fig. 5 SEC-LLS chromatograms of the samples.((a) Laser light scattering photometry and RI for NTWP-II and (b) molar mass distribution analysis of NTWP-II). 


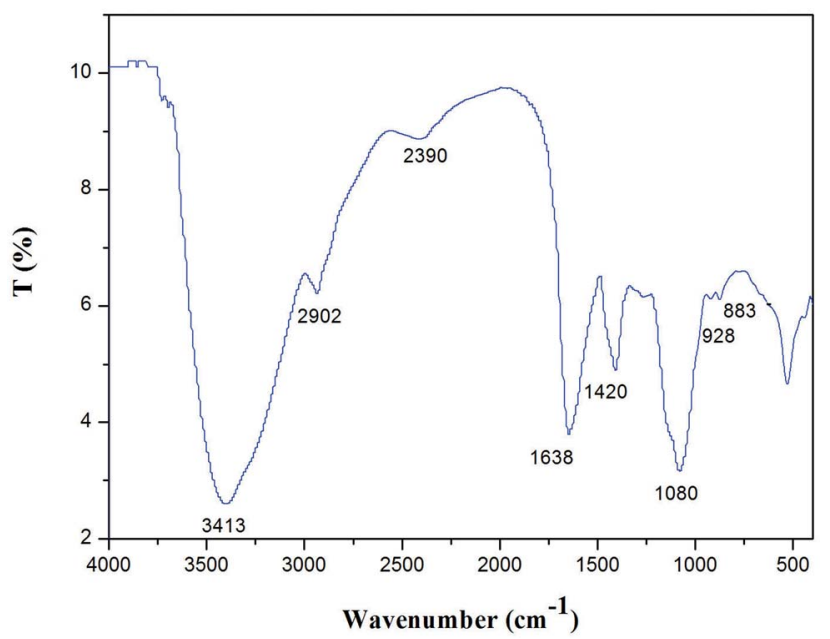

Fig. 6 FT-IR spectroscopy of NTWP-II.

To ensure the predicted result was not biased toward the practical value, experimental rechecking was performed using this deduced optimal condition. A mean value of $14.01 \pm 0.11 \%$ $(n=3)$, obtained from real experiments, demonstrated the validation of the RSM model. The good correlation between experimental and predicted values confirmed that the response model was accurate and adequate for the extraction of NTWP. The validation result revealed that there was no significant difference between experimental and predicted values, suggesting that the response model was adequate for reflecting the expected optimization.

\subsection{Isolation and purification of polysaccharide fraction NTWP}

NTWP solution ( $5 \mathrm{mg} \mathrm{mL}^{-1}$ ) was loaded into a DEAE cellulose52 column equilibrated with a linear gradient elution of $\mathrm{NaCl}$ from $0 \mathrm{M}$ to $1.0 \mathrm{M}$. Two independent elution peaks (NTWP-I $24.11 \%$ and NTWP-II $64.57 \%$, Fig. 3A) detected at $490 \mathrm{~nm}$ by the phenol-sulfuric acid assay were obtained.

NTWP-I consisted of glucose and NTWP-II consisted of rhamnose, mannose, glucose, galactose and arabinose by GC-MS. The next research focused on NTWP-II. Thus, only the NTWP-II fraction was collected for the subsequent purification and antioxidant activity assays. The NTWP-II fraction was collected, dialyzed, concentrated, and loaded into a Sephadex G-200 column. The fraction produced a single elution peak (Fig. 3B).

\subsection{Chemical composition of NTWP-II}

NTWP-II isolated from the fruit of Nitraria tangutorum Bobr. by a series of purification procedures, including water extraction, ethanol sedimentation, deproteinization, and dialysis. The total sugar content of NTWP-II separated by Sephadex G-200 column was $94.3 \pm 3.42 \%$ and was poor in protein $(2.22 \pm 0.34 \%)$. Through acid hydrolysis, NTWP-II was subjected to GC-MS analysis and the results were shown in Fig. 4. As can be seen in Fig. 4a, NTWP-II was composed of rhamnose, arabinose, mannose, glucose and galactose with the molar ratio of $1.14: 2.5: 3.00: 2.69: 5.28$. These results didn't show a good correlation with those reported by $\mathrm{Ni}$ et al. They found that NTWP was composed of mannose, rhamnose, glucuronicacid, galacturonic acid, glucose, galactose and arabinose with approximate molar ratios of 3.9 : 1.8 : 0.2 : 3.3 : 70.6 : 7.6 : 13.1. This result might be due to regional difference.

\subsection{Molecular weight determination}

The SEC-LLS chromatogram patterns of NTWP-II were shown in Fig. 5a and b. The chromatograms of NTWP-II exhibited a single peak indicating that there was no aggregation and the homogeneity of the purified samples. The molecular weight of NTWPII was determined by SEC-LLS. The weight average molar mass $\left(M_{\mathrm{w}}\right)$, polydispersity (PD, $\left.M_{\mathrm{w}} / M_{\mathrm{n}}\right)$ and $z$-average radius of gyration $\left(R_{\mathrm{z}}\right)$ were $2.29 \times 10^{5}, 1.32,15.22$, respectively.

\subsection{FT-IR analysis of NTWP-II}

NTWP-II was characterized by FT-IR spectroscopy as shown in Fig. 6. The infrared spectra showed strong and wide stretching peak around $3413 \mathrm{~cm}^{-1}$ for $\mathrm{O}-\mathrm{H}$ stretching vibrations as well as a weak absorption peak at $2902 \mathrm{~cm}^{-1}$ for $\mathrm{C}-\mathrm{H}$ stretching vibrations. A strong absorption peak at $1618 \mathrm{~cm}^{-1}$ was attributed to asymmetric and symmetric stretching of the carboxylate anion group (COO), indicating NTWP-II be acidic polysaccharides. Each particular polysaccharide has a specific band in the 1000$1200 \mathrm{~cm}^{-1}$ region. This region was dominated by ring vibrations overlapped with stretching vibrations of $(\mathrm{C}-\mathrm{OH})$ side groups and the (C-O-C) glycosidic band vibration. The absorptions at $1072.99 \mathrm{~cm}^{-1}$ indicated a pyranose form of sugar. ${ }^{16,17}$ A specific band in the $1080 \mathrm{~cm}^{-1}$ region indicated a pyranose form of sugar. ${ }^{17,18}$ In the anomeric region $\left(950-700 \mathrm{~cm}^{-1}\right)$, the spectrum exhibited the characteristic absorption at $883 \mathrm{~cm}^{-1}$ due to the presence of mannose. ${ }^{12}$ This showed a good correlation with monosaccharide composition. These results indicated that NTWP-II possessed typical absorption peak of polysaccharides.

\subsection{Nuclear magnetic resonance (NMR) spectroscopy analysis}

NMR spectroscopy could provide detailed structural information including the monosaccharide composition, $\alpha$ - or $\beta$ anomeric configurations, linkage patterns, and sequences of the sugar units. Signals of NTWP-II in $1 \mathrm{D}^{1} \mathrm{H}$ and ${ }^{13} \mathrm{C}$ NMR and 2D NMR (HMQC, HMBC, COSY, NOESY and TOCSY) spectra were assigned as completely as possible, based on the monosaccharide analysis and chemical shifts reported in the literature. ${ }^{20,21}$ The ${ }^{1} \mathrm{H}$ and ${ }^{13} \mathrm{C}$ NMR spectra of NTWP-II were shown in Fig. 7a and b. The ${ }^{1} \mathrm{H}$ NMR spectrum was crowded in a narrow region ranging from 3 to $5 \mathrm{ppm}$ which was typical of polysaccharides, and this confirmed the presence of many similar sugar residues. ${ }^{22,23}$

${ }^{1} \mathrm{H}$ NMR spectrum (Fig. 7b) NTWP-II contained five signals at $\delta 5.19,5.02,4.43,5.23$ and 5.03 ppm for the anomeric protons, indicating for five residues which were designated as A, B, C, D and E, respectively, present in NTWP-II. The anomeric protons of residues A (5.19 ppm), B (5.02 ppm), D (5.23 ppm) and E (5.03 $\mathrm{ppm}$ ) has the chemical shifts larger than $5.0 \mathrm{ppm}$ and very small $J$-coupling constants of ${ }^{3} J_{\mathrm{H}-1, \mathrm{H}-2}$ (the values of ${ }^{3} J_{\mathrm{H}-1, \mathrm{H}-2}$ was 2.17, 
a
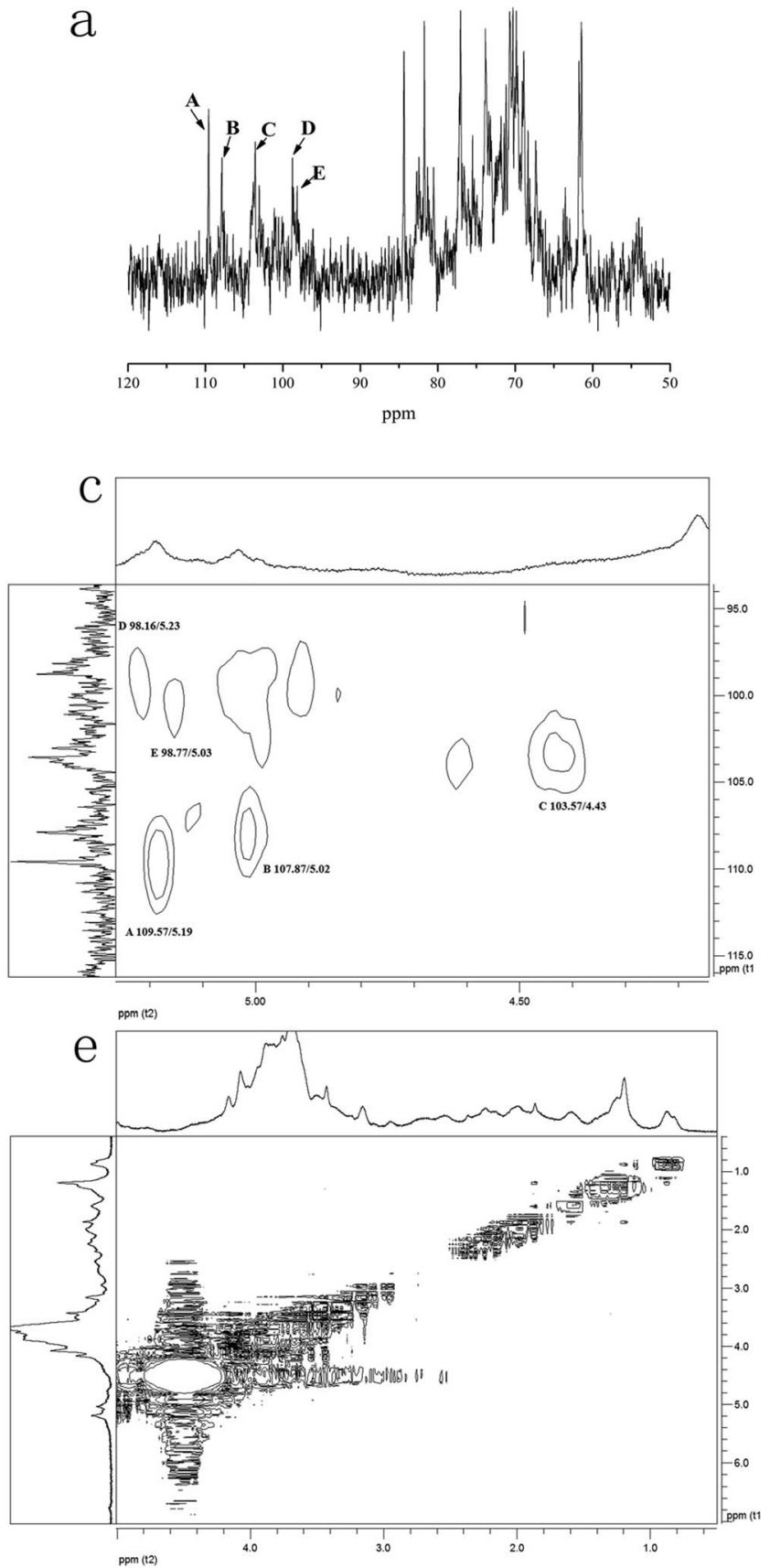

b
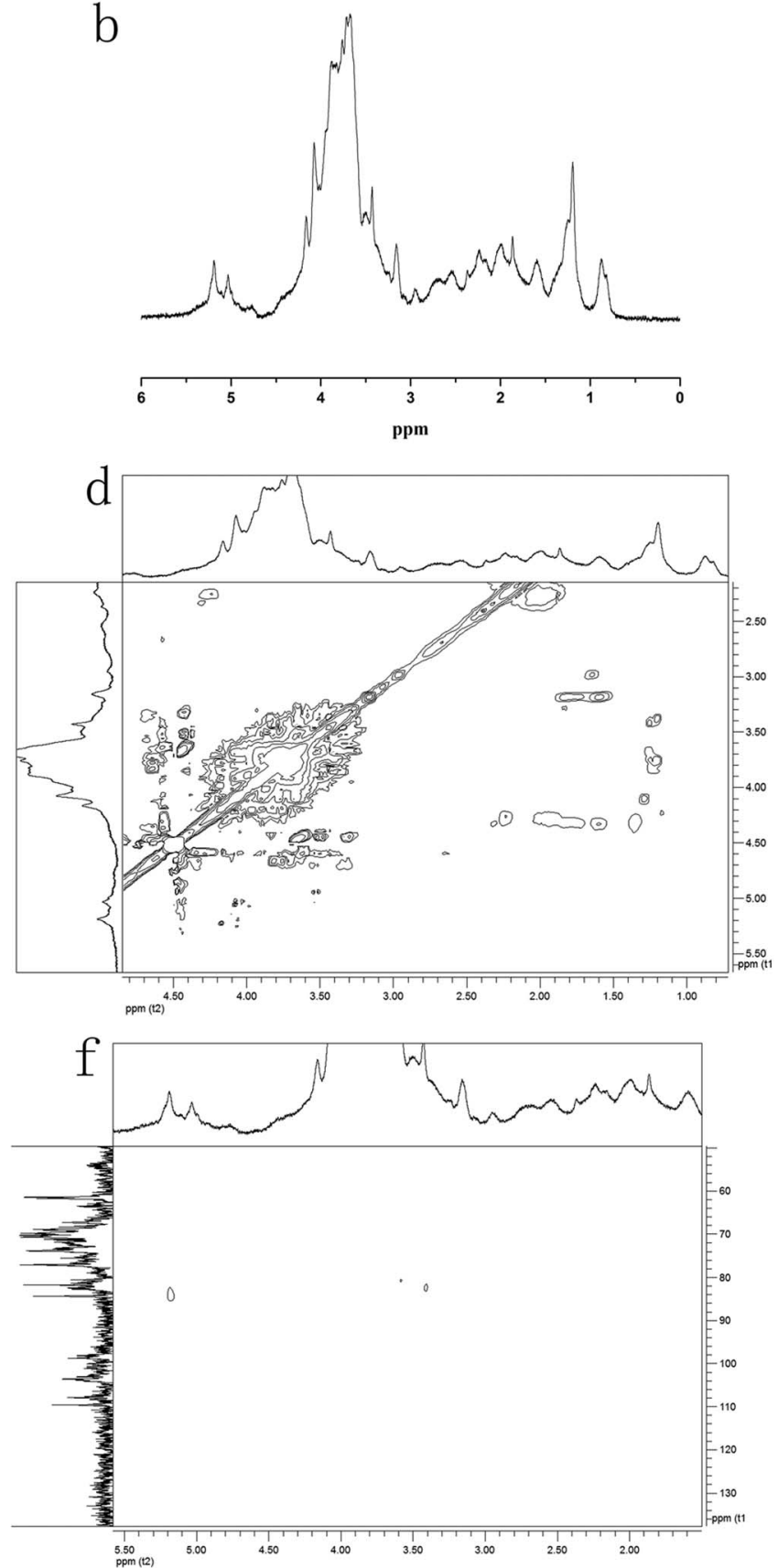

Fig. 7 NMR spectra of the sulfated polysaccharide NTWP-II. (a) ${ }^{1} \mathrm{H}$ NMR spectrum; (b) ${ }^{13} \mathrm{C}$ NMR spectrum; (c) ${ }^{1} \mathrm{H}-{ }^{13} \mathrm{C} \mathrm{HMQC} \mathrm{spectrum;} \mathrm{(d)}{ }^{1} \mathrm{H}-{ }^{1} \mathrm{H}$ TOCSY spectrum; (e) ${ }^{1} \mathrm{H}_{-}{ }^{13} \mathrm{C}$ HMBC spectrum; and (f) ${ }^{1} \mathrm{H}-{ }^{1} \mathrm{H}$ NOESY.

$2.23,0.86$ and $3.84 \mathrm{~Hz}$, respectively), suggesting that residues A, $\mathrm{B}, \mathrm{D}$ and $\mathrm{E}$ were $\alpha$-linked. Meanwhile the anomeric proton of residue $\mathrm{C}$ has the chemical shift smaller than $5.0 \mathrm{ppm}$ and suggested residue $\mathrm{C}$ being $\beta$-linked. ${ }^{20,21,24}$ The $1 \mathrm{D}{ }^{13} \mathrm{C}$ NMR spectra (Fig. 7a) showed five signals in the anomeric region ranging from $\delta 90$ to $\delta 110$. These results were consistent with the fact that the NTWP-II consisted of five types of monosaccharides. Furthermore, the cross-peaks of $\delta 109.57 / 5.19$, 107.87/5.02, 103.57/4.43, 98.16/5.23, and 98.77/5.03 ppm were observed in 2D HMQC NMR spectrum (Fig. 7c), indicating that the anomeric carbon signals at $\delta 109.57,107.87,103.57,98.16$ and $98.77 \mathrm{ppm}$ corresponded to the anomeric proton signals at $\delta 5.19,5.02,4.43,5.23$ and 5.03 , respectively.

In TOCSY and HMQC spectrum (Fig. 7d and c), $\delta 61.44 \mathrm{ppm}$ and $\delta 68.35 \mathrm{ppm}$ were attributed to no-substitution of C-6 and weak substitution of C-6. Based on the chemical shifts of $\mathrm{H}, \mathrm{C}$ and ${ }^{3} \mathrm{~J}_{\mathrm{H}-1, \mathrm{H}-2}$ of residues $\mathrm{C}$, residues $\mathrm{C}$ was identified as $\beta$-galactose. According to the molar ratio of monosaccharide, signal intensity of ${ }^{13} \mathrm{C}$ spectrum (C-1 $\delta 103.57 \mathrm{ppm}$ and C-3 $\delta 75.50 \mathrm{ppm}$ ) and weak substitution of $\mathrm{C}-6$, it was concluded that residue $\mathrm{C}$ was $(1 \rightarrow 3)-\beta$-galactose and the main sugar units of backbone. The anomeric carbon signals at $\delta 109.57$ of residues A indicated that the position of the glycosidic 
linkage of A was C-1. Based on standard monosaccharide and former research, residues A was identified as $\alpha$-arabinose, because the chemical shifts of $\mathrm{C}-1(\delta 97.6 \mathrm{ppm})$ in $\alpha$-arabinose was larger than in $\beta$-arabinose ( $\delta 93.4 \mathrm{ppm})$. The chemical shifts of C-4 $(\delta 71.19$ ppm) down low field confirmed the substitution at position C-3. Therefore, we inferred that residue $A$ was $(1 \rightarrow 4)$ - $\alpha$-arabinose. Compared with standard monosaccharide, residues $\mathrm{E}$ was identified as $(1 \rightarrow)$ - $\alpha$-mannose, residues D and B were identified as $(1 \rightarrow)-\alpha-$ glucose and $(\rightarrow 6)-\alpha$-rhamnose, respectively from the chemical shifts of residues D (C-6 $\delta 63.53 \mathrm{ppm})$ and B (C-1 $\delta 107.87 \mathrm{ppm})$.

In NOESY spectrum (Fig. 7f), the crosspeaks between $\mathrm{C} \mathrm{H}-3$ and $\mathrm{E} \mathrm{H}-1, \mathrm{~A} \mathrm{H}-1$ and $\mathrm{C} \mathrm{H}-3$ indicated that the presence of the bond $(1 \rightarrow 3)$ between $\mathrm{C}$ and $\mathrm{E}, \mathrm{A}$ and $\mathrm{C}$. In HMBC spectrum (Fig. 7e), some crosspeaks were observed between $\mathrm{H}-1$ and ${ }^{13} \mathrm{C}$ peak in different residues: E C-1 and $\mathrm{B}$ H-2, and E C-1 and D H-6. These HMBC data revealed the existence of two sequences in the structure: $\mathrm{E}(1 \rightarrow 2) \mathrm{B}, \mathrm{E}(1 \rightarrow 6) \mathrm{D}$.
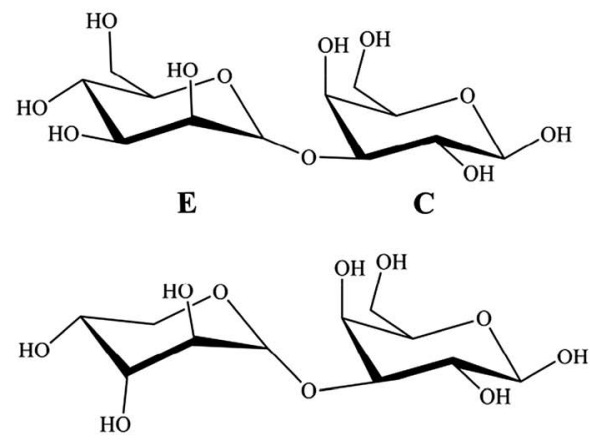

A

C

According to previous studies of NTP-II, $(1 \rightarrow 3)$ - $\beta$-galactose was the main sugar units of backbone and linked with $\alpha$-mannose, $\alpha$ arabinose and $\beta$-galactose by the glycosidic linkage of $(1 \rightarrow 3)$ and $(\rightarrow 1)$ - $\alpha$-glucose of the side chain was obtained. It was proposed that the residue sequence in the repeating unit was as:

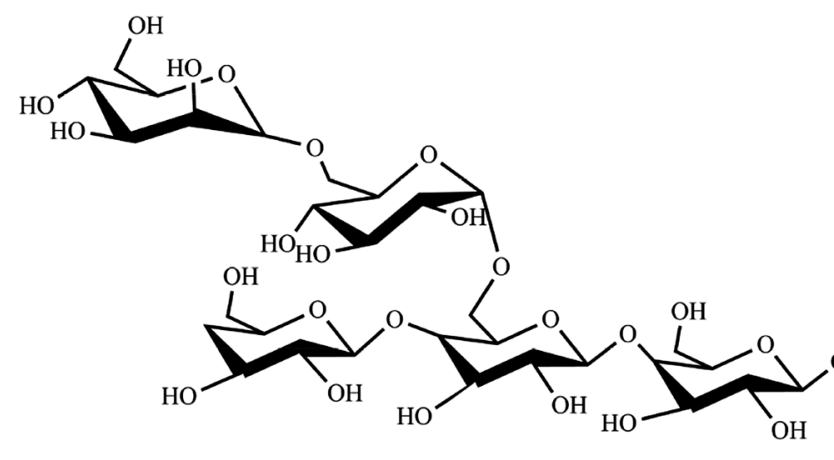

\subsection{Antioxidant activities in vitro of NTP-II}

DPPH radical was a stable free radical, of which alcohol solution has characteristic absorption maximum at $517 \mathrm{~nm}$ and used to evaluate the free radical scavenging activities of natural compounds. When DPPH ethanol solution was reduced, absorbance was decreased and the solution changed from purple to light yellow. ${ }^{17}$ The reduction extent of absorbance reflects the hydrogen or electron donating abilities of antioxidants. ${ }^{25}$ As shown in Fig. 8a, at the range from $0.02 \mathrm{mg} \mathrm{mL}^{-1}$ to $1 \mathrm{mg} \mathrm{mL} \mathrm{m}^{-1}$, the color significantly became light with the increase of NTWP-II concentrations. Beyond the concentration of $1 \mathrm{mg} \mathrm{mL} \mathrm{m}^{-1}$, the trend was not significant. However, the scavenging effect of NTWP-II on DPPH radical was lower than that of BHT. Previous studies demonstrated that the antioxidant activity of polysaccharides has been attributed to their
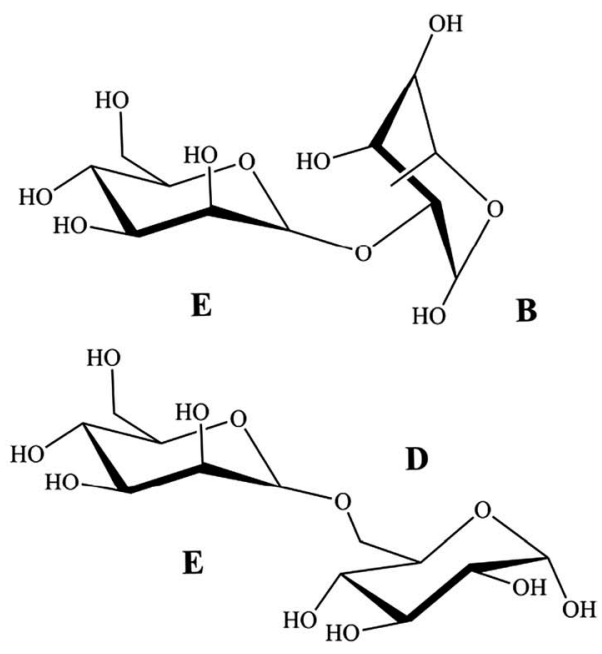

composition and structural features. ${ }^{26}$ The results implied that NTWP-II could have stronger ability to donate electron or hydrogen. ${ }^{25}$

Hydroxyl radical could cause severe damage to adjacent biomolecules or cell death. ${ }^{\mathbf{1 9}}$ For hydroxyl radical, there were two types of antioxidation mechanism: one suppresses the

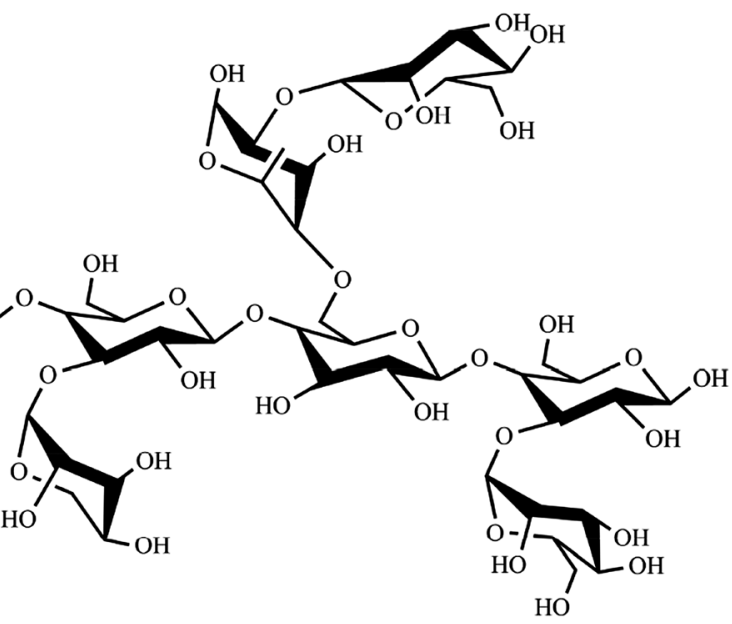



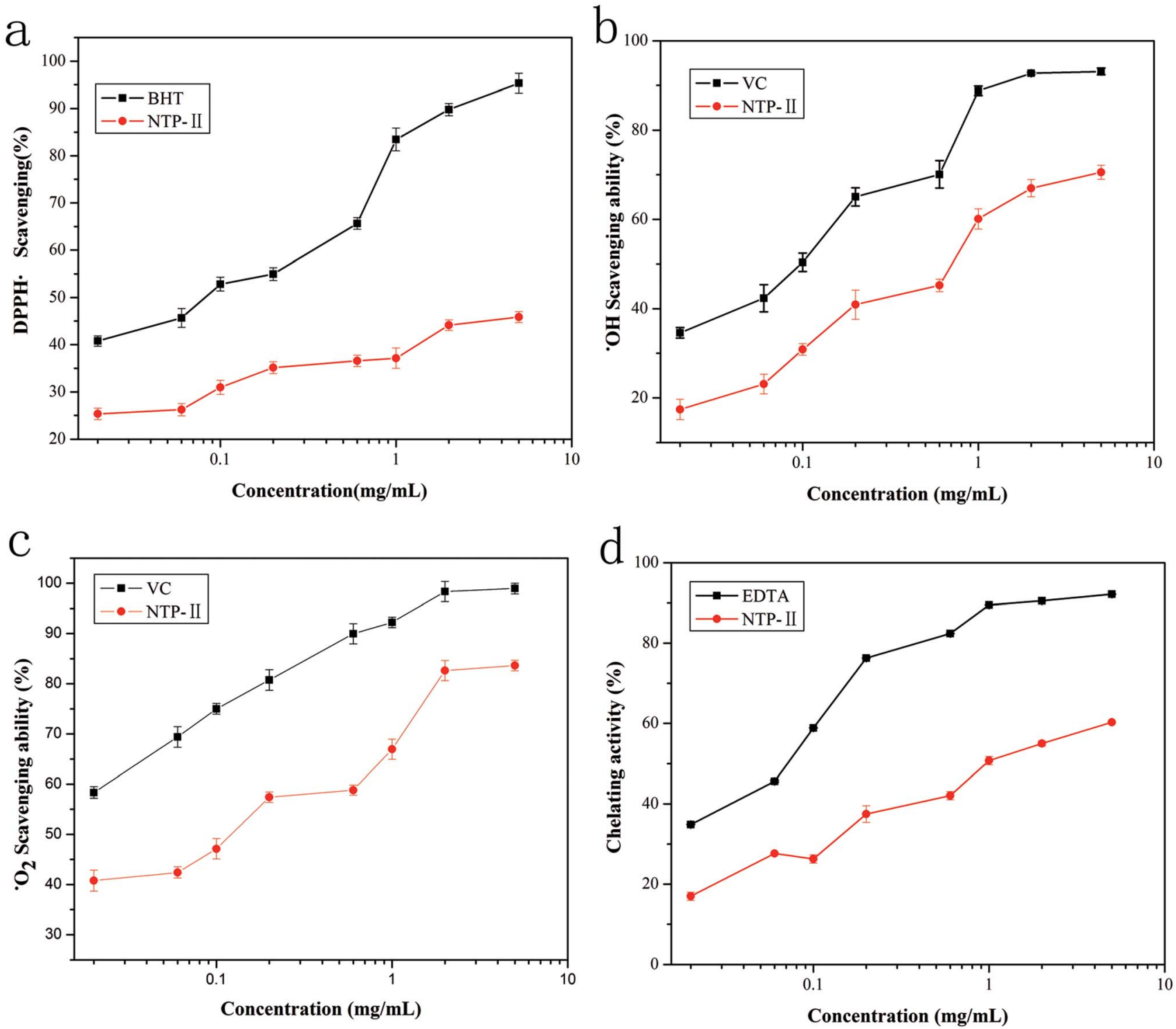

Fig. 8 Antioxidant activity of NTWP-II at different concentrations ((a) scavenging activity of NTWP-II and BHT at different concentrations on DPPH radicals, (b) scavenging activity of NTWP- $\|_{E}$ and $V_{C}$ at different concentrations on hydroxyl radicals, (c) scavenging effects of NTWP-II and $V_{C}$ at different concentrations on superoxide radical, (d) chelating activity of NTWP-II and EDTA at different concentrations on ferrous ion). Data are shown as mean $\pm \mathrm{SD}(n=3)$.

generation of the hydroxyl radical, and the other scavenges the hydroxyl radicals generated. Thus, it forms a stable radical to terminate the radical chain. It has been found that the antioxidant activities of polysaccharides are affected by various factors such as chemical contents, molecular mass and structure. ${ }^{27}$ The scavenging effect on hydroxyl radical of NTWP-II was shown in Fig. 8b. The hydroxyl radical, known to be generated through the Fenton reaction in this system, was scavenged by samples. For NTWP-II, the effects of scavenging hydroxyl radicals were in a concentration-dependent manner from $0.04-3 \mathrm{mg} \mathrm{mL}^{-1}$. The $\mathrm{IC}_{50}$ value of NTWP-II was $0.82 \mathrm{mg} \mathrm{mL}^{-1}$. According to the previous study, polysaccharides with moderate molecular weights have been found to have strong antioxidant activity. In this study, NTWP-II showed strong hydroxyl radical scavenging activity. This might be attributed to moderate molecular weight and high contents of hydrogen. However, the relationships between antioxidant activity and physicochemical property or structural features of the polysaccharide have not been comprehensively understood. ${ }^{28}$

Superoxide anion radicals are weak oxidants and are thus not harmful to the body. However, its combination with hydroxyl molecules may damage DNA and other biomolecules. Scavenging effects of NTWP-II and $\mathrm{V}_{\mathrm{C}}$ on superoxide radical were shown in Fig. 8c. For these two samples, scavenging activities of NTWP-II and $\mathrm{V}_{\mathrm{C}}$ followed a dose-dependent manner at all tested concentrations. The scavenging effects significantly increased with increasing concentration from $0.04 \mathrm{mg} \mathrm{mL}^{-1}$ to $3.0 \mathrm{mg} \mathrm{mL}{ }^{-1}$ and $\mathrm{IC}_{50}$ values for scavenging superoxide radical 
was $0.35 \mathrm{mg} \mathrm{mL}^{-1}$. The results indicated that NTWP-II has a noticeable superoxide radical scavenging activity.

Chelation is an important biological process, as iron is an essential metallic element for respiration, oxygen transport, and the activity of many enzymes for metabolism. Among the transition metals, $\mathrm{Fe}^{2+}$ is known as the most powerful prooxidant due to its high reactivity, which accelerates the reactions of oxidation through the Fenton reaction. ${ }^{29}$ The ferrous ion chelating activity of NTWP-II at different concentrations was shown in Fig. 8d and compared with EDTA in the equivalent concentration as a positive control. The metal chelating ability was recognized as a correlative activity to antioxidant. From Fig. 8d, we knew the chelating activity of NTWP-II exhibited a much weaker metal chelating ability. The chelating rate for NTWP-II was only $57.3 \%$ even at $5.0 \mathrm{mg} \mathrm{mL}{ }^{-1}$, while that of EDTA was $60.1 \%$ at $0.6 \mathrm{mg} \mathrm{mL}^{-1}$. Metal chelation activity reduces the concentrations of the transition metals, thereby catalysing lipid peroxidation and thus reducing oxidative reactions. The reducing power results revealed that NTWP-II can act as electron donor compound, react with free radicals and convert them to more stable products terminating, therefore, reactions of the radical chain.

\section{Conclusion}

In order to increase the extraction yield of polysaccharides form the fruit of Nitraria tangutorum Bobr. The optimal extraction conditions with hot water extraction were optimized by BBD. The optimum variables given by BBD were as follows: extraction time $7 \mathrm{~h}$, extraction temperature $60{ }^{\circ} \mathrm{C}$, liquid-solid ratio $15: 1$. Under these conditions, the experimental yield was obtained as $14.01 \pm 0.11 \%(n=3)$, which corresponded with the predicted value well. The purified homogeneous polysaccharides NTWP-II was successfully obtained by DEAE-cellulose column and Sephadex G-200 column chromatography. In addition, chemical analysis indicated that the weight average molar mass $\left(M_{\mathrm{w}}\right)$, polydispersity (PD, $\left.M_{\mathrm{w}} / M_{\mathrm{n}}\right)$ and $z$-average radius of gyration $\left(R_{\mathrm{z}}\right)$ were $2.29 \times 10^{5}, 1.32,15.22$ by SEC-LLS. Monosaccharide analysis revealed that NTWP-II was composed of rhamnose, arabinose, mannose, glucose and galactose with the molar ratio of $1.14: 2.5: 3.00: 2.69: 5.28$. Based on the analysis of monosaccharide composition, $1 \mathrm{D}$ and $2 \mathrm{D} \mathrm{NMR}$, the backbone structure of NTWP-II consisted mainly of $(1 \rightarrow 3)$ - $\beta$-galactose. Besides, and linked with $\alpha$-mannose, $\alpha$-arabinose and $\beta$-galactose by the $(1 \rightarrow 3)$ glycosidic linkage and $(\rightarrow 1)$ - $\alpha$-glucose of the side chain was obtained. NTWP-II exhibited positive radical scavenging activities against DPPH radical, superoxide anion and hydroxyl radicals, and metal chelating ability in vitro. Thus, NTWP-II could be explored as a natural antioxidant food ingredient. These might further provide theoretical basis for the widely application of NTWP-II in medicine and health care products.

\section{Conflicts of interest}

There are no conflicts to declare.

\section{Acknowledgements}

We gratefully acknowledge the financial supported by Science \& Technological Department of Gansu Province, P. R. China (17JR5RA152), and Sheng Tong Sheng Scientific Research Foundation from Gansu Agricultural University University (GSAU-STS-1531).

\section{References}

1 Y. F. Wang, Z. W. Yang and X. L. Wei, Int. J. Biol. Macromol., 2012, 50, 558-564.

2 K. D. Jaya and S. R. Jaya, Asian Pac. J. Trop. Med., 2012, 362366.

3 H. L. Wang, Y. R. Suo and X. Y. Wang, Sep. Purif. Technol., 2007, 56, 371-377.

4 J. Zheng, H. Li and C. X. Ding, Food Res. Int., 2011, 44, 20412046.

5 H. Y. Li, W. J. Mao and X. L. Zhang, Carbohydr. Polym., 2011, 85, 394-400.

6 J. N. Duan, I. D. Williams and C. T. Che, Tetrahedron Lett., 1999, 40, 2593-2596.

7 B. P. L. Liu, E. Y. Y. Chong and F. W. K. Cheung, Biochem. Pharmacol., 2005, 70, 287-299.

8 W. H. Ni, T. T. Gao and H. L. Wang, J. Ethnopharmacol., 2013, 150, 529-535.

9 S. François, G. Corinne and A. A. Haji, Nitrariaceae, J. Ethnopharmacol., 2012, 141, 629-634.

10 L. Zhang, S. S. Ji and S. Wang, Sci. Technol. Food Ind., 2013, 34, 170-174.

11 B. T. Zhao, J. Zhang and X. Guo, Food Hydrocolloids, 2013, 31, 346-356.

12 J. Chen, T. Zhang and B. Jiang, Carbohydr. Polym., 2012, 87, 40-45.

13 Z. G. Liu, L. J. Mei and Q. L. Wang, LWT-Food Sci. Technol., 2014, 56, 168-174.

14 T. Saeed and G. Mitra, Carbohydr. Polym., 2014, 112, 396403.

15 Y. P. Wang, Y. Liu and Y. H. Hu, Carbohydr. Polym., 2014, 111, 324-332.

16 Z. Ying, X. X. Han and J. R. Li, Food Chem., 2011, 127, 12731279.

17 W. C. Zeng, Z. Zhang and G. Hong, Carbohydr. Polym., 2012, 89, 694-700.

18 Q. P. Xiong, X. Li and R. Z. Zhou, Carbohydr. Polym., 2014, 108, 247-256.

19 Z. Y. Wang, Y. Y. Zhao and T. T. Su, Carbohydr. Polym., 2010, 115, 25-31.

20 I. Peres, S. Rocha and M. C. Pereira, Carbohydr. Polym., 2015, 82, 861-866.

21 H. Y. Li, W. Y. Mao and X. L. Zhang, Carbohydr. Polym., 2011, 85, 394-400.

22 M. Kohno, S. Suzuki and T. Kanaya, Carbohydr. Polym., 2009, 77, 351-357.

23 D. Pan, L. Q. Wang and C. H. Chen, Food Chem., 2012, 135, 1097-1103. 
24 X. H. Qi, W. J. Mao and Y. Gao, Carbohydr. Polym., 2012, 90, 27 I. Khemakhem, O. Abdelhedi, I. Trigui, M. A. Ayadi and 1804-1810.

M. Bouaziz, Int. J. Biol. Macromol., 2018, 106, 425-432.

25 B. B. Ren, C. Chen, C. Li, X. Fu, L. J. You and R. H. Liu, 28 X. Y. Liu, Y. X. Chen, L. X. Wu, X. Q. Wu, Y. F. Huang and Carbohydr. Polym., 2017, 173, 192-201.

26 C. Li, X. S. Li, L. J. You, X. Fua and R. H. Liu, Carbohydr. 29 L. Wang, H. M. Liu and G. Y. Qin, Food Chem., 2017, 234, Polym., 2017, 155, 261-270. 314-322. 